\title{
Tubulointerstitial Macrophage Accumulation is Regulated by Sequentially Expressed Osteopontin and Macrophage Colony-Stimulating Factor: Implication for the Role of Atorvastatin
}

\author{
Shaojiang Tian, Guohua Ding, Ruhan Jia, and Guili Chu \\ Division of Nephrology, Renmin Hospital of Wuhan University, Wuhan, Hubei 430060, China
}

Received 26 September 2005; Accepted 3 November 2005

Infiltration and local proliferation are known factors that contribute to tubulointerstitial macrophage accumulation. This study explored the time course of these two contributors' roles as tubulointerstitial inflammation and fibrosis progressing, and evaluated the mechanisms of the protective effect of atorvastatin. Unilateral ureteral obstructive (UUO) rats were treated with atorvastatin $(10 \mathrm{mg} / \mathrm{Kg})$ or vehicle. Expression of osteopontin (OPN) and macrophage colony-stimulating factor (M-CSF) was evaluated by RT-PCR and immunohistochemistry. Immunohistochemistry staining of ED1 was used to assess macrophage accumulation in interstitium. Histological evaluation was performed to semiquantify tubulointerstitial fibrosis. The results showed that on day 3 after UUO operation, OPN expression significantly increased and positively correlated with the number of the interstitial ED $1^{+}$ cells, while on day 10, M-CSF expression upregulated and correlated with interstitial ED1 ${ }^{+}$cells. In atorvastatin treatment group, the increments of these two factors were attenuated significantly at the two time points, respectively. ED $1^{+}$cell accumulation and fibrosis also ameliorated in the treatment group. For all the samples of UUO and treatment group on day 10 , ED1 $1^{+}$cells also correlated with interstitial fibrosis scores. The results suggest that OPN may induce the early macrophage/monocyte infiltration and M-CSF may play an important role in regulating macrophage accumulation in later stage of UUO nephropathy. Statin treatment decreases interstitial inflammation and fibrosis, and this renoprotective effect may be mediated by downregulating the expression of OPN and M-CSF.

Copyright ( 2006 Shaojiang Tian et al. This is an open access article distributed under the Creative Commons Attribution License, which permits unrestricted use, distribution, and reproduction in any medium, provided the original work is properly cited.

\section{INTRODUCTION}

The chronic inflammation characterized by macrophage accumulation in glomeruli and the interstitium is a common feature in most types of glomerulonephritis. Tubulointerstitial macrophage accumulation in particular correlates with kidney disease progression [1]. Indeed, studies have shown that interstitial macrophage accumulation is predictive of disease progression in severe forms of human and experimental glomerulonephritis [2-4]. The accumulated macrophage may directly or indirectly be involved in tubulointerstitial fibrosis [5], which is the hallmark of irreversible chronic kidney injury. So, the study investigating the mechanisms of macrophage accumulation may shed a light on prevention of chronic kidney disease.

Studies have shown that interstitial macrophage accumulation is due to the results of monocyte/macrophage (M/M) infiltration and local proliferation [6]. Recent study has revealed that osteopontin (OPN) plays an important role in chemotaxis on $\mathrm{M} / \mathrm{M}$ infiltration in a rat model of anti-GBM glomerulonephritis [7], and macrophage colonystimulating factor (M-CSF) is a critical factor that induces strong local macrophage proliferation [8, 9]. Although the function of these two cytokines has been identified, which one at which time plays a predominant role remains unclear. Ophascharoensuk et al reported that macrophage influx was less in OPN-/- mice compared to $\mathrm{OPN}+/+$ mice in early stage (day 4 and day 7) in unilateral ureteral obstructive (UUO) nephropathy, but not in later stage (day 14) [10]. Le Meur et al have also found that following UUO, kidney MCSF mRNA increased in association with local macrophage proliferation in later stage (days 5 and 10). Anti-c-fms (antibody to receptor of M-CSF) treatment caused a minor inhibition of monocyte recruitment at day 1 , but reduced macrophage accumulation by $75 \%$ at day 10 [11]. Those studies implicate that different cytokines may be responsible 
for the macrophage accumulation in different phases. However, the study investigating the time course of these two cytokines' expression in a same group of patients/animals is still lacking, so we cannot get an integral interpretation about the time order of these two contributors' roles.

Chronic unilateral ureteral obstruction is a well-characterized experimental model of renal injury leading to tubulointerstitial inflammation and fibrosis [12]. This is because it is normotensive, nonproteinuric, nonhyperlipidemic, and without any apparent immune or toxic renal insult. Studies have shown the prominent $\mathrm{M} / \mathrm{M}$ accumulation in UUO kidney, so we chose UUO rat as our interstitial inflammation and fibrosis model. Meanwhile, recent studies have revealed that statin can suppress the accumulation of $\mathrm{M} / \mathrm{M}$ in this experimental kidney disease $[13,14]$, so we testify the protective effect of atorvastatin and try to investigate the mechanisms of this effect in UUO nephropathy.

\section{MATERIALS AND METHOD}

\section{Animals and reagents}

Adult male Sprague-Dawley rats (180-220 g) were obtained from Experimental Animal Breeding Center of Medical College of Wuhan University. Monoclonal antibodies used were mouse anti-rat ED1 (Serotec Co), mouse anti-rat OPN (National Health Research Institution, USA), and goat anti-rat M-CSF (Santa Cruz Biotech). The primers for OPN, MCSF, and GAPDH were produced by TaKaRa Co. SPkit was the production of Zymed Co. Atorvastatin was provided by Pfizer Pharmaceutical Ltd.

\section{Experimental design}

All the 42 rats were randomly divided into 3 groups as follows: (1) sham-operated group; (2) UUO; (3) UUO+ atorvastatin. The general procedure of rat UUO operation is the same as previously described [15]. After general anesthesia by muscular injection of ketaminum ( $60 \mathrm{mg} / \mathrm{kg}$ body weight), all the animals underwent left proximal ureteral ligation. The left ureter was identified through a suprapubic incision and was ligated with 4.0 silk at two points, and then cut between the two points. Sham operation was done without ureteral ligation and cut. The left kidneys were harvested from the animals at day 3 or 10 after ureteral obstruction operation. At each time point, 7 rats were sacrificed in each group.

In UUO+ atorvastatin group, rats were orally administered atorvastatin $(10 \mathrm{mg} / \mathrm{kg}$ body weight per day) from three days before the UUO operation to the day of sacrifice. For all animals, before removing the kidney, blood samples were obtained from heart, and then centrifuged at $3000 \mathrm{rpm}$ and serum was stored at $-70^{\circ} \mathrm{C}$ for later use.

\section{Morphology}

Renal tissue at sacrifice was fixed in $10 \%$ formalin and embedded in paraffin. For evaluating tubular lesions, sections were stained with PAS reagent. Tubular lesions, characterized by tubular dilation and epithelial desquamation with interstitial expansions, were graded according to the extent of cortical involvement on a scale from 0 to 4 [16]: $0=$ normal; $1=$ involvement of less than $25 \%$ of the cortex; $2=$ involvement up to $25 \%$ to $50 \%$ of the cortex; $3=$ involvement up to $50 \%$ to $75 \%$ of the cortex; $4=$ extensive damage involving more than $75 \%$ of the cortex. Then they were expressed as tubular injury scores. An observer blinded to the origin of the sections, examined tubulointerstitial fields adjacent to an arbitrary glomerulus at $\times 200$. Sections were also stained with Masson trichrome identifying collagen fibers (in blue). Histological assessment of collagen deposition was determined by the point-counting method using a $10 \times 10$ grid [17]. A minimum of 10 high power $(\times 400)$ fields were assessed per animal and results expressed as \% total interstital cortical area, excluding glomeruli, blood vessels, and periglomerular and perivascular areas.

\section{Immunohistochemistry}

Sections of formalin-fixed, paraffin-embeded tissue were dewaxed, rehydrated, microwaved, and stained using a standard three-layer method as recommended by SP kit. When measuring OPN, the first antibody was mouse anti-rat OPN antibody; when examining the M-CSF expression, the first antibody was goat anti-rat M-CSF antibody; and when measuring macrophage accumulation, the first antibody was mouse anti-rat ED1 antibody. The bound peroxidase was developed with diaminobenzidine to produce a brown colour followed by a blue nuclear haematoxylin counterstain.

\section{Quantitation of immunohistochemistry}

The OPN and M-CSF immunostaining was measured by evaluating the labeling index in tubules of the kidney with a computer-assisted image analyzer system (HPIAS-1000) and expressed by integral optical density (IOD). The number of interstitial $\mathrm{ED}^{+}$cells was counted in 100 consecutive highpower $(\times 400)$ fields and expressed as cells per HP.

\section{Reverse transcriptase-polymerase chain reaction}

Total RNA was extracted from kidney tissue using RNAzol BTM (Protech, Inc, USA) according to the manufacturer's instructions. Reverse transcription of $1 \mu \mathrm{g}$ RNA was performed using $100 \mathrm{pmol}$ random OligA primers (Amersham Pharmacia Biotech, Uppsala, Sweden) and $300 \mathrm{U} \mathrm{Su-}$ perScript RNAse $H$ (GIBCO BRL) with the mixture of $5 \mu \mathrm{l}$ buffer, $10 \mu \mathrm{mol} \mathrm{dNTP}, 0.1 \mathrm{mmol}$ DTT, and $60 \mathrm{U}$ RNAsin (Boehringer Mannheim, Mannheim, Germany) at $42^{\circ} \mathrm{C}$ for $1 \mathrm{~h}$. PCR was performed using the following primers: OPN: forward primer $5^{\prime}$-GAT GAG TCC TTC ACT GCC AGCA; reverse primer $3{ }^{\prime}$-CGA TAG CAT CCG ACC GCT CTG (amplifying a 418 bp fragment); M-CSF: sense-AGT GAG GGA TTT TTG ACC CAG; antisense-AGA TGA ACC ATC CGT CTT CTC (amplifying a 234 bp fragment); GAPDH: sense-ACC ATG GAG AAG GCT, antisense-AGT GTA GCC CAG GAT (amplifying a $522 \mathrm{bp}$ fragment). PCR of $2 \mu \mathrm{l}$ of the cDNA was performed with $0.025 \mathrm{U} / \mu \mathrm{l}$ Taq polymerase (GIBCO BRL), 2 mM MgCl2, 0.2 mM dNTP, PCR buffer, and 


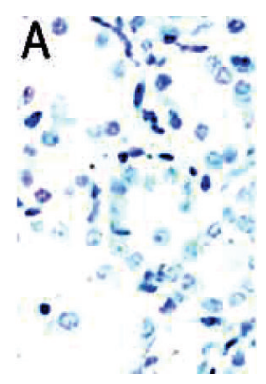

(a)

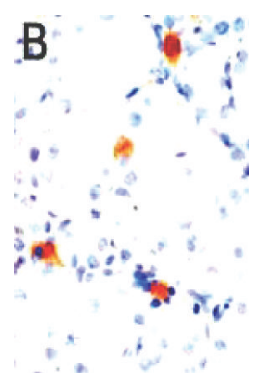

(b)

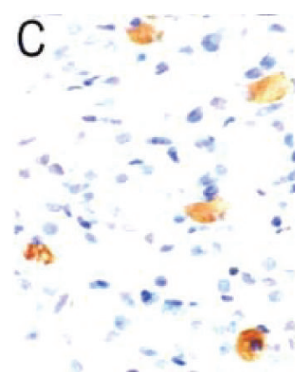

(c)

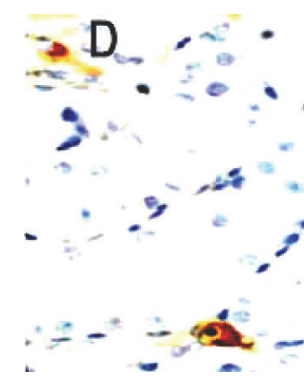

(d)

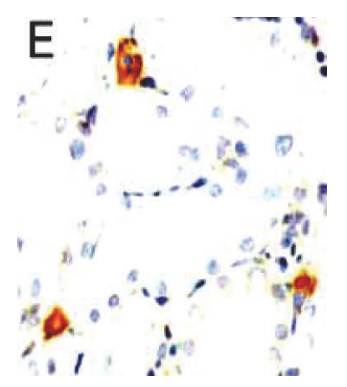

(e)

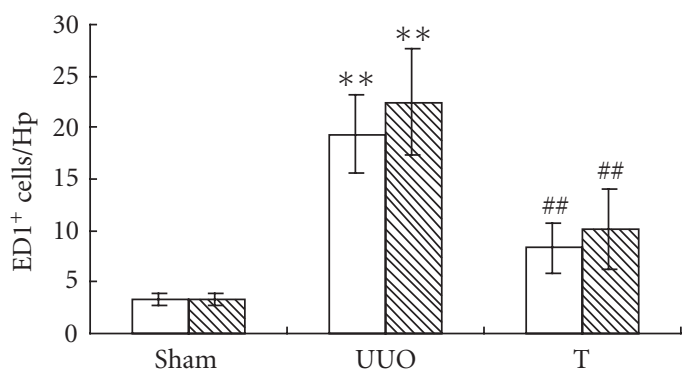

$\square$ Day 3

$\mathbb{\$}$ Day 10

Figure 1: ED1+ cell accumulation at different time points in three groups $(\times 400)$. Sections were stained using a standard three-layer method as recommended by SP kit. Macrophage accumulation was measured by immunohistochemistry and quantified by the number of ED1 $1^{+}$cells/HP: (a) sham group, (b) UUO on day 3, (c) UUO on day 10, (d) treatment group on day 3, and (e) treatment group on day $10{ }^{* *}: P<.01$ versus sham group of the same time point. \#\#: $P<.01$ versus UUO group of the same time point.

$0.5 \mu \mathrm{M}$ of each primer in a final volume of $50 \mu \mathrm{l}$ in a DNA thermocycler 480 (Perkin Elmer, Norwalk, Conn, USA). After 3 min at $94^{\circ} \mathrm{C}$, PCR was conducted for 30 cycles (pilot studies were performed to ensure that the reaction was within the linear phase) using the following conditions: 455 of denaturation at $94^{\circ} \mathrm{C}, 1 \mathrm{~min}$ of annealing at $55 / 57^{\circ} \mathrm{C}$, and 1 min of extension at $72^{\circ} \mathrm{C}$, followed by a final extension for 5 min at $72^{\circ} \mathrm{C}$ and cooling to $4^{\circ} \mathrm{C}$.

The PCR products were separated by electrophoresis on a $1.5 \%$ agarose gel (GIBCO BRL). Following staining with ethidium bromide, the gels were photographed and digitized by using a scanner (Lacie Silver Scanner for Macintosh) and the DeskScan software (Adobe PhotoShop). The image was inverted before performing densitometric analysis by using National Institues of Health Image 1.6 software. A ratio of the intensity between OPN and GAPDH or M-CSF and GAPDH was calculated.

\section{Measurement of serum lipids}

The serum lipid was tested in our hospital laboratory department. The parameters include total cholesterol, total triglycerol, and HDL-cholesterol.

\section{Statistical analysis}

Results are present as the mean $\pm \mathrm{SD}$. The number of macrophage, expression of OPN and M-CSF at different time points in three groups, was analyzed by one-way analysis of variance (ANOVA) followed by $q$ test using the SPSS for windows 10.0. In addition, the correlations between macrophage accumulation and cytokines staining or fibrosis index were performed by the Spearman's rank correlation coefficient. Statistical significance was defined as $P<.05$.

\section{RESULTS}

\section{Macrophage accumulation}

As illustrated in Figure 1, few macrophages were observed in sham-operated rat kidney. There was a significant increase in the number of interstitial $\mathrm{ED1}^{+}$cells on day 3 in UUO group and more $\mathrm{ED} 1^{+}$accumulation on day 10. In UUO+ atorvastatin-treated group, interstitial $\mathrm{ED} 1^{+}$macrophage accumulation was markedly decreased on day 3 and day 10 when comparing with UUO animals at the same time points, respectively. 


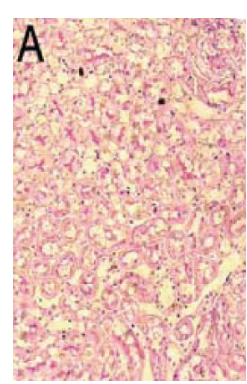

(a)

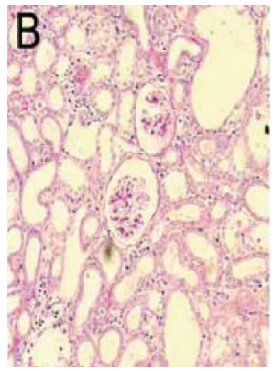

(b)

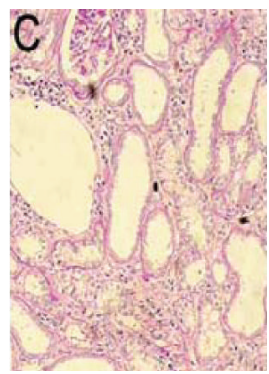

(c)

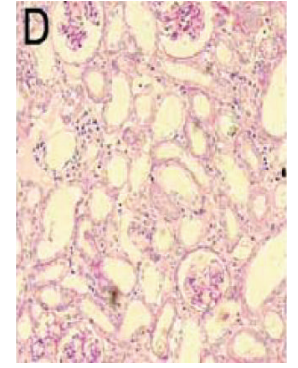

(d)

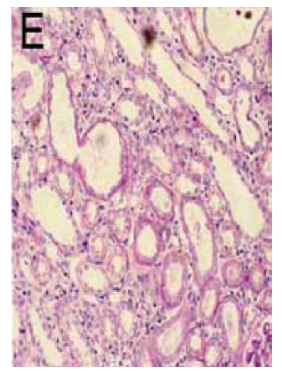

(e)

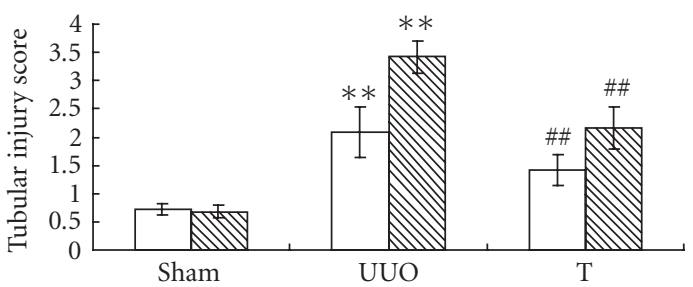

ㅁ Day 3

\$ Day 10

Figure 2: Tubular injury score at different time points in three groups $(\times 100)$. Sections were stained with PAS regent. Tubular lesions were graded according to the extent of cortical damage: (a) sham group, (b) UUO on day 3, (c) UUO on day 10, (d) treatment group on day 3 , and (e) treatment group on day $10{ }^{* *}: P<.01$ versus sham group of the same time point. \#\#: $P<.01$ versus UUO group of the same time point.

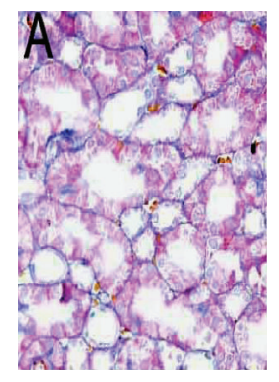

(a)

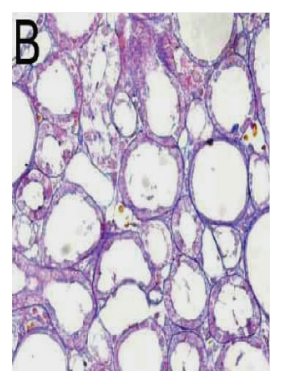

(b)

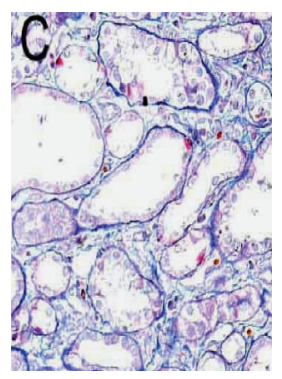

(c)

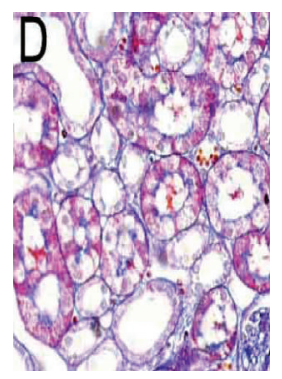

(d)

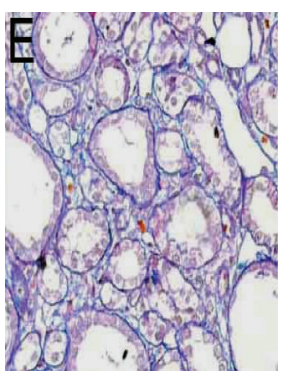

(e)

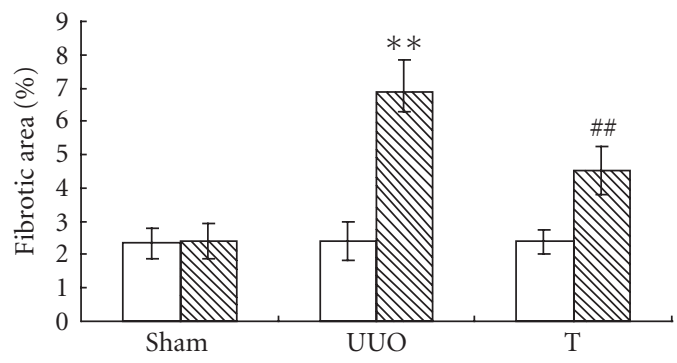

Day 3

\& Day 10

FIgURe 3: Tubulointerstitial fibrosis at different time points in three groups $(\times 200)$. Sections were stained with Masson trichrom identifying collagen fibers. Collagen deposition was determined by the point-counting method: (a) sham group, (b) UUO on day 3, (c) UUO on day 10, (d) treatment group on day 3 , and (e) treatment group on day $10{ }^{* *}: P<.01$ versus sham group of the same time point. \#\#: $P<.01$ versus UUO group of the same time point. 


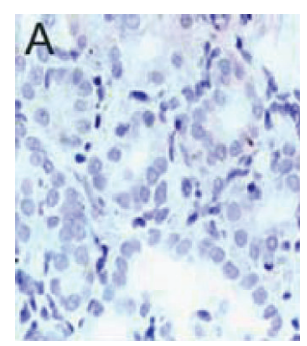

(a)

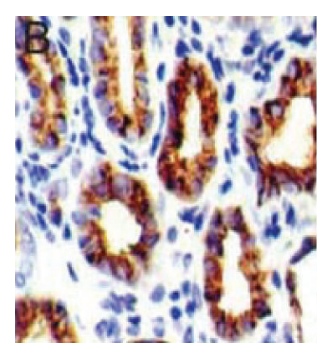

(b)

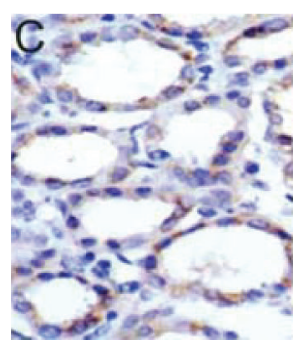

(c)

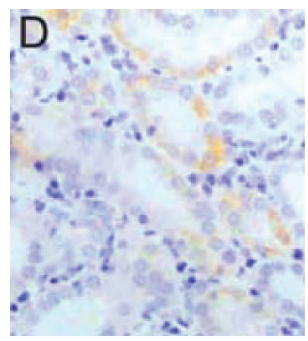

(d)

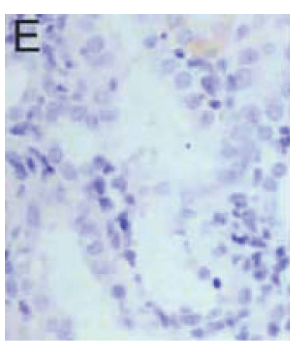

(e)

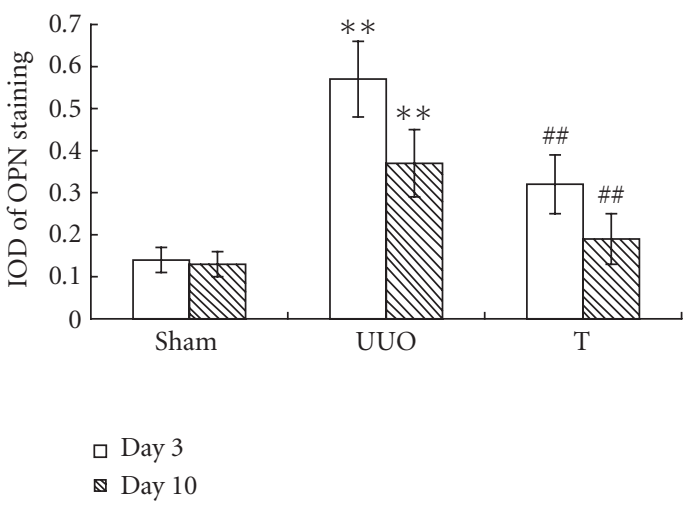

FIGURE 4: Staining of OPN at different time points in different groups $(\times 200)$. Using antibody against OPN, sections were stained by immunohistochemistry. Labeling index was expressed by integral optical density (IOD): (a) sham group, (b) UUO on day 3, (c) UUO on day 10, (d) treatment group on day 3 , and (e) treatment group on day 10 . $^{* *}: P<.01$ versus sham group of the same time point. \#\#: $P<.01$ versus UUO group of the same time point.

\section{Tubulointerstitial injury and fibrosis and correlation with ED1 ${ }^{+}$cell accumulation}

There was marked tubular damage on day 3 and more prominently on day 10 after UUO operation, atorvastatin treatment reduces tubular injury both on day 3 and on day 10 (as shown in Figure 2). To assess tubulointerstitial fibrosis, Masson trichrome staining was performed. The results indicated significantly higher fibrosis score on day 10 in UUO group. The atorvastatin treatment attenuated interstitial fibrosis at that time point (as shown in Figure 3). For all samples of UUO and treated group on day 10, the number of $\mathrm{ED}^{+}{ }^{+}$interstitial cells significantly correlated with the index of interstitial fibrosis detected by Masson trichrome staining $(r=0.58, P<.01)$.

\section{OPN and M-CSF expression and correlation with macrophage accumulation}

The induction of UUO nephropathy led to significantly increased tubular expression of OPN on day 3, but this increase attenuated significantly on day 10 . The expression of M-CSF did not upregulate on day 3 but increased and reached statistical significance on day 10 . In atorvastatin-treated group, the OPN expression on day 3 and the M-CSF expression on day 10 were significantly ameliorated, respectively, when comparing with UUO group (results are shown in Figures 4 and 5). The reverse transcriptase-polymerase chain reaction (RT-PCR) analysis indicated that the mRNA of OPN on day 3 in UUO group increased by about 13-fold comparing with that of sham group, but decreased to about 9-fold on day 10 (as shown in Figure 6). The expression of M-CSF mRNA was statistically identical to sham group on day 3 and dramatically increased by about 7-fold on day 10 in the UUO group (data shown in Figure 7). When taking all the samples of UUO and UUO+ atorvastatin group on day 3 for correlation analysis, there was a significant positive correlation between the $\mathrm{ED}^{+}$cells accumulation and the staining of OPN $(r=0.71, P<.01)$. When analyzing all samples of these two groups on day 10 , the $\mathrm{ED} 1^{+}$cell accumulation was positively correlated with the staining of M-CSF $(r=0.82, P<.01)$.

\section{Serum lipid}

Serum lipid level of all the 3 groups is shown in Table 1. As it demonstrated, there was no significant deviation on any time points among these groups.

\section{DISCUSSION}

The present study demonstrated that in early stage of UUO nephropathy, OPN expression increased and correlated with 


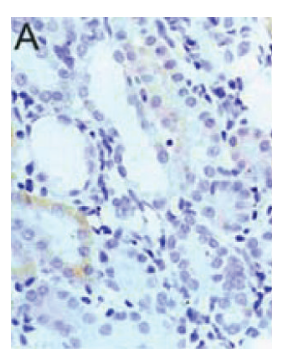

(a)

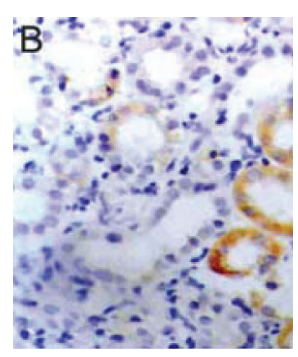

(b)

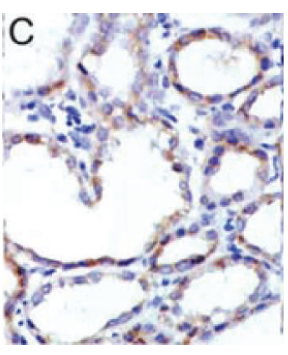

(c)

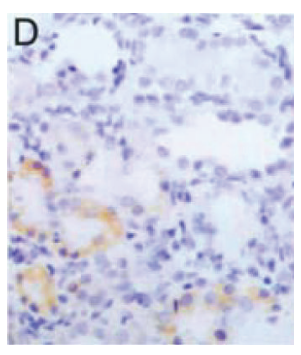

(d)

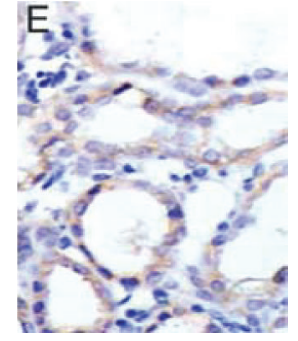

(e)

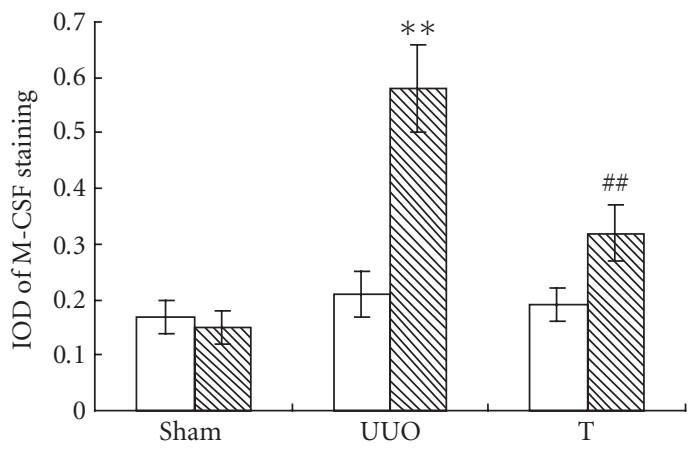

$\square$ Day 3

$\mathbb{D}$ Day 10

FIGURE 5: Staining of M-CSF at different time points in different groups by immunohistochemistry $(\times 200)$. Using antibody against M-CSF, sections were stained by immunohistochemistry. Labeling index was expressed by integral optical density (IOD): (a) sham group, (b) UUO on day 3, (c) UUO on day 10, (d) treatment group on day 3, and (e) treatment group on day 10. ${ }^{* *}: P<.01$ versus sham group of the same time point. \#\#: $P<.01$ versus UUO group of the same time point.

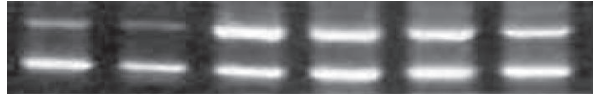

OPN GAPDH

(a)

(b)

(c)

(d)

(e)

(f)

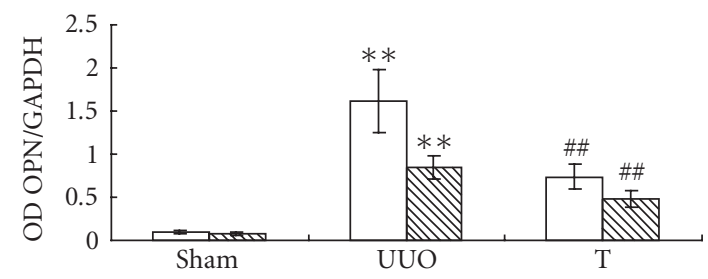

口 Day 3

ه Day 10

FIGURE 6: Expression of OPN at different time points in different groups by RT-PCR. Total RNA was extracted from kidney tissue at the indicated time and reverse-transcribed to cDNA. cDNA was subject to PCR, and OPN was amplified to 418 bp fragments. GAPDH was used as internal control (a) on day 3 in sham group, (b) on day 10 in sham group, (c) on day 3 in UUO group, (d) on day 10 in UUO group, (e) on day 3 in treatment group, and (f) on day 10 in treatment group. ${ }^{* *}: P<.01$ versus sham group of the same time point. \#\#: $P<.01$ versus UUO group of the same time point. 

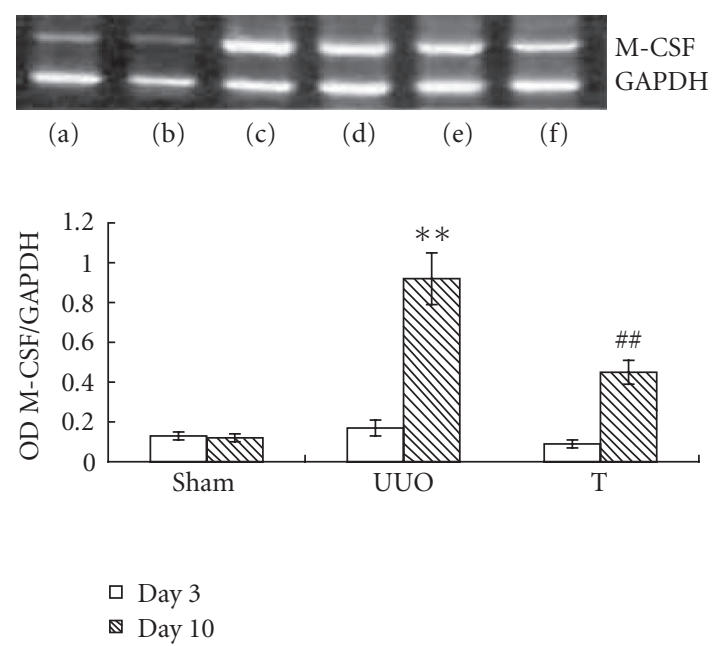

FIGURE 7: Expression of M-CSF at different time points in different groups by RT-PCR. Total RNA was extracted from kidney tissue at the indicated time and reverse-transcribed to cDNA. cDNA was subject to PCR, and M-CSF was amplified to $234 \mathrm{bp}$ fragments. GAPDH was used as internal control (a) on day 3 in sham group, (b) on day 10 in sham group, (c) on day 3 in UUO group, (d) on day 10 in UUO group; (e) on day 3 in treatment group, and (f) on day 10 in treatment group. ${ }^{* *}: P<.01$ versus sham group of the same time point. \#\#: $P<.01$ versus UUO group of the same time point.

TABLE 1: Serum lipid level at different time points of different groups.

\begin{tabular}{|c|c|c|c|c|c|c|}
\hline & \multicolumn{2}{|c|}{ Sham } & \multicolumn{2}{|c|}{ UUO } & \multicolumn{2}{|c|}{ Treated } \\
\hline & Day 3 & Day 10 & Day 3 & Day 10 & Day 3 & Day 10 \\
\hline T-ch $(\mathrm{mmol} / \mathrm{L})$ & $1.53 \pm 0.21$ & $1.64 \pm 0.78$ & $1.57 \pm 0.49$ & $1.48 \pm 0.36$ & $1.62 \pm 0.59$ & $1.54 \pm 0.28$ \\
\hline $\mathrm{T}-\mathrm{G}(\mathrm{mmol} / \mathrm{L})$ & $0.76 \pm 0.37$ & $0.69 \pm 0.45$ & $0.72 \pm 0.29$ & $0.72 \pm 0.11$ & $0.75 \pm 0.23$ & $0.76 \pm 0.33$ \\
\hline HDL-C (mmol/L) & $1.11 \pm 0.35$ & $1.06 \pm 0.52$ & $1.16 \pm 0.49$ & $1.20 \pm 0.66$ & $1.19 \pm 0.32$ & $1.09 \pm 0.41$ \\
\hline
\end{tabular}

interstitial macrophage accumulation, while in later stage, M-CSF expression increased and correlated with interstitial macrophage accumulation. Atorvastatin treatment ameliorated the increments of these two cytokines' expression at two time points, respectively, and also reduced the interstitial macrophage accumulation and fibrosis.

Numerous studies have investigated the role of OPN and M-CSF in tubulointerstitial macrophage accumulation [79, 18]. Using OPN knockout mice, Persy et al verified that OPN was a critical factor for interstitial macrophage accumulation [19]. Meanwhile, Lenda et al also confirmed that macrophage proliferation was reduced in M-CSF deficient mice [20]. On the contrary to the abundant studies in testing those cytokines' role, only few reports evaluated the time course of these two factors' roles. An integral and comprehensive explanation that temporally considered these two inflammatory mediators is still lacking. The present study identified, to our knowledge for the first time, the pattern of these two cytokines' expression in UUO nephropathy, and provided strong evidence for our postulation that it is the different cytokines that play a predominant role at different time points in macrophage accumulation in UUO nephropathy. Our findings make us reason that OPN and M-CSF are sequentially expressed in tubular cells and regulated interstitial macrophage accumulation in different phases.

Our study also indicated that macrophage accumulation correlated with interstitial fibrosis. Although tubulointerstitial inflammation may not be the prerequisite of the onset of interstitial fibrosis [21], and fibrosis cannot be reversed by agents that suppress inflammatory cell activation alone [22], taken together with other studies, our study support that interstitial inflammation may be involved in and accelerate this process. Recent study has also revealed that chemokine receptor antagonist reduce interstitial inflammation and fibrosis $[3,23]$. All those reports underline the recognition that macrophage accumulation could be considered as a major component involved in the tubulointerstitial fibrosis.

Statins (HMG-CoA reductase inhibitor) have been shown to inhibit macrophage accumulation in tubulointerstitium independent of their cholesterol-lowering effects [24]. This study demonstrated that atorvastatin reduced the number of macrophage on day 3 and on day 10 after UUO operation through downregulating the expression of OPN and M-CSF independent of cholesterol-lowering effects. We suppose that this may be the mechanistic insight that explains how atorvastatin exerts this anti-inflammation effect. 
Studies also have shown that atorvastatin downregulates the expression of adhesion molecule in endothelial cells [25], which is also related to macrophage accumulation. In UUO nephropathy, atorvastatin reduces OPN expression which may also be related to its inhibiting effect on angiotensin II [26], because studies have shown that angiotensin II is a potent inducer of OPN [18]. On the other hand, atorvastatin also can inhibit NF-kappa B activation [13]. This effect may be related to its role in downregulating M-CSF expression [27]. But, all these presumptions need more studies to investigate because of the complicated interaction of these cytokines in vivo.

In our study, atorvastatin significantly reduced interstitial fibrosis. This effect may be related to its role in reducing macrophage accumulation as this study identified, but it may also be related to its pleiotrophic effect on other fibrogenic factors. As we discussed above, macrophage may not be the only contributor to interstitial fibrosis, blockade of the angiotensin II type 2 receptor can also suppress interstitial fibrosis without affecting interstitial macrophage accumulation [28]. As statin holds the capacity of inhibiting angiotensin II [13], we guess that atorvastatin can also reduce fibrosis independent of its role in reducing macrophage accumulation.

In summary, this study has demonstrated that OPN and M-CSF expression was upregulated in tubular cells in rat UUO nephropathy in different phases, and correlated with local macrophage accumulation at related time points. Atorvastatin may decrease macrophage accumulation through inhibiting OPN and M-CSF expression, and finally ameliorate the interstitial fibrosis.

\section{ACKNOWLEDGMENTS}

The authors are grateful to Dr Layuan Xiong and Dr Hongxia Yang, Laboratory Center of Nephrology, for their expert technical assistance. They thank Dr Shiquan Liu for many helpful discussions.

\section{REFERENCES}

[1] Eddy AA. Progression in chronic kidney disease. Advances in Chronic Kidney Disease. 2005;12(4):353-365.

[2] Schwarz A, Mengel M, Gwinner W, et al. Risk factors for chronic allograft nephropathy after renal transplantation: a protocol biopsy study. Kidney International. 2005;67(1):341348.

[3] Anders HJ, Belemezova E, Eis V, et al. Late onset of treatment with a chemokine receptor CCR1 antagonist prevents progression of lupus nephritis in MRL-Fas(lpr) mice. Journal of the American Society of Nephrology: JASN. 2004;15(6):1504-1513.

[4] Wu Q, Jinde K, Endoh M, Sakai H. Clinical significance of costimulatory molecules CD80/CD86 expression in IgA nephropathy. Kidney International. 2004;65(3):888-896.

[5] Young BA, Burdmann EA, Johnson RJ, et al. Cellular proliferation and macrophage influx precede interstitial fibrosis in cyclosporine nephrotoxicity. Kidney International. 1995;48(2):439-448.

[6] Lan HY, Nikolic-Paterson DJ, Mu W, Atkins RC. Local macrophage proliferation in the progression of glomerular and tubulointerstitial injury in rat anti-GBM glomerulonephritis. Kidney International. 1995;48(3):753-760.

[7] Panzer U, Thaiss F, Zahner G, et al. Monocyte chemoattractant protein-1 and osteopontin differentially regulate monocytes recruitment in experimental glomerulonephritis. Kidney International. 2001;59(5):1762-1769.

[8] Isbel NM, Hill PA, Foti R, et al. Tubules are the major site of M-CSF production in experimental kidney disease: correlation with local macrophage proliferation. Kidney International. 2001;60(2):614-625.

[9] Isbel NM, Nikolic-Paterson DJ, Hill PA, Dowling J, Atkins RC. Local macrophage proliferation correlates with increased renal M-CSF expression in human glomerulonephritis. Nephrology Dialysis Transplantation. 2001;16(8):1638-1647.

[10] Ophascharoensuk V, Giachelli CM, Gordon K, et al. Obstructive uropathy in the mouse: role of osteopontin in interstitial fibrosis and apoptosis. Kidney International. 1999;56(2):571580.

[11] Le Meur Y, Tesch GH, Hill PA, et al. Macrophage accumulation at a site of renal inflammation is dependent on the M-CSF/cfms pathway. Journal of Leukocyte Biology. 2002;72(3):530537.

[12] Klahr S, Morrissey JJ. Obstructive nephropathy and renal fibrosis. American Journal of Physiology-Renal Physiology. 2002;283(5):F861-F875.

[13] Massy ZA, Guijarro C. Statins: effects beyond cholesterol lowering. Nephrology Dialysis Transplantation. 2001;16(9):17381741 .

[14] Mizuguchi Y, Miyajima A, Kosaka T, Asano T, Asano T, Hayakawa M. Atorvastatin ameliorates renal tissue damage in unilateral ureteral obstruction. The Journal of Urology. 2004;172(6 pt 1):2456-2459.

[15] Edgtton KL, Gow RM, Kelly DJ, Carmeliet P, Kitching AR. Plasmin is not protective in experimental renal interstitial fibrosis. Kidney International. 2004;66(1):68-76.

[16] Mizuno S, Matsumoto K, Nakamura T. Hepatocyte growth factor suppresses interstitial fibrosis in a mouse model of obstructive nephropathy. Kidney International. 2001;59(4):13041314.

[17] Isaka $Y$, Tsujie $M$, Ando $Y$, et al. Transforming growth factor$\beta 1$ antisense oligodeoxynucleotides block interstitial fibrosis in unilateral ureteral obstruction. Kidney International. 2000;58(5):1885-1892.

[18] Xie Y, Sakatsume M, Nishi S, Narita I, Arakawa M, Gejyo F. Expression, roles, receptors, and regulation of osteopontin in the kidney. Kidney International. 2001;60(5):1645-1657.

[19] Persy VP, Verhulst A, Ysebaert DK, De Greef KE, De Broe ME. Reduced postischemic macrophage infiltration and interstitial fibrosis in osteopontin knockout mice. Kidney International. 2003;63(2):543-553.

[20] Lenda DM, Kikawada E, Stanley ER, Kelley VR. Reduced macrophage recruitment, proliferation, and activation in colony-stimulating factor-1-deficient mice results in decreased tubular apoptosis during renal inflammation. The Journal of Immunology. 2003;170(6):3254-3262.

[21] Lavaud S, Poirier B, Mandet C, et al. Inflammation is probably not a prerequisite for renal interstitial fibrosis in normoglycemic obese rats. American Journal of Physiology-Renal Physiology. 2001;280(4):F683-F694.

[22] Lange-Sperandio B, Forbes MS, Thornhill B, Okusa MD, Linden J, Chevalier RL. A(2A) adenosine receptor agonist and $\operatorname{PDE}(4)$ inhibition delays inflammation but fails to reduce injury in experimental obstructive nephropathy. Nephron. Experimental Nephrology. 2005;100(3):e113-e123. 
[23] Vielhauer V, Berning E, Eis V, et al. CCR1 blockade reduces interstitial inflammation and fibrosis in mice with glomerulosclerosis and nephrotic syndrome. Kidney International. 2004;66(6):2264-2278.

[24] Vieira JM Jr, Mantovani E, Rodrigues LT, et al. Simvastatin attenuates renal inflammation, tubular transdifferentiation and interstitial fibrosis in rats with unilateral ureteral obstruction. Nephrology Dialysis Transplantation. 2005;20(8):1582-1591.

[25] Serrano CV Jr, Yoshida VM, Venturinelli ML, et al. Effect of simvastatin on monocyte adhesion molecule expression in patients with hypercholesterolemia. Atherosclerosis. 2001;157(2):505-512.

[26] Park JK, Muller DN, Mervaala EM, et al. Cerivastatin prevents angiotensin II-induced renal injury independent of blood pressure-and cholesterol-lowering effects. Kidney International. 2000;58(4):1420-1430.

[27] Wardle EN. Nuclear factor $\kappa B$ for the nephrologist. Nephrology Dialysis Transplantation. 2001;16(9):1764-1768.

[28] Morrissey JJ, Klahr S. Effect of AT2 receptor blockade on the pathogenesis of renal fibrosis. American Journal of Physiology—Renal Physiology. 1999;276(1 pt 2):F39-F45. 


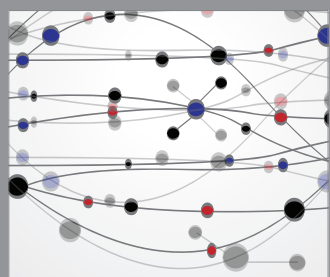

The Scientific World Journal
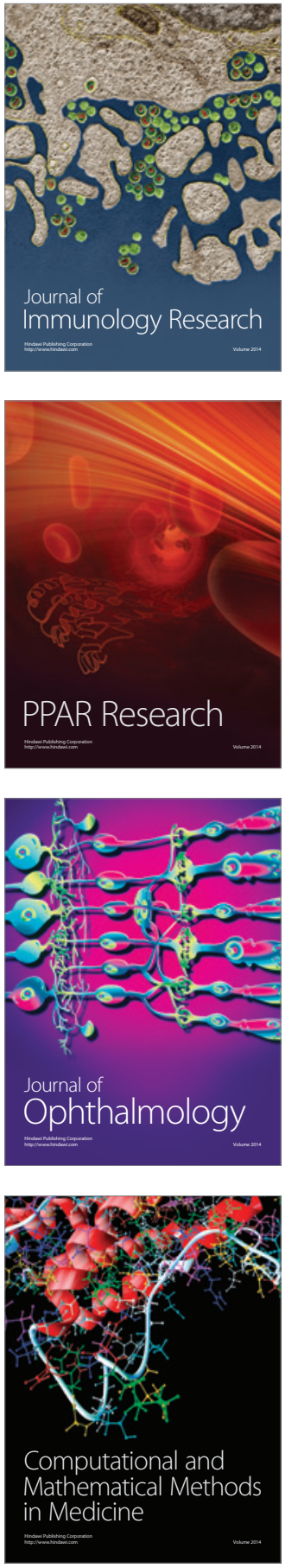

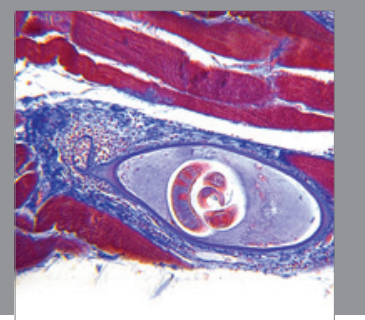

Gastroenterology

Research and Practice
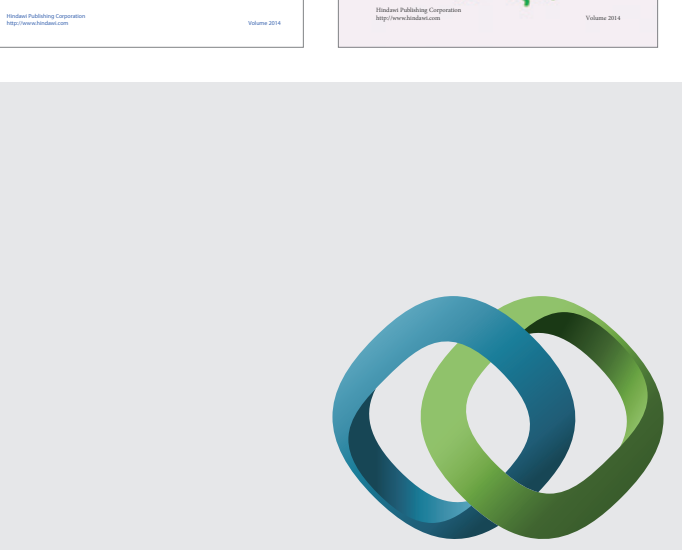

\section{Hindawi}

Submit your manuscripts at

http://www.hindawi.com
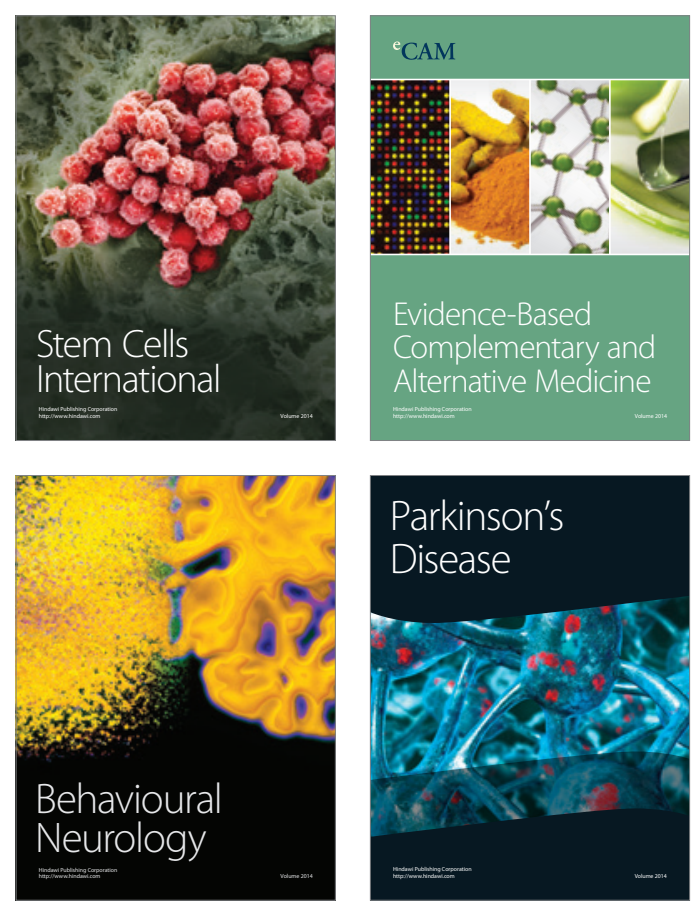

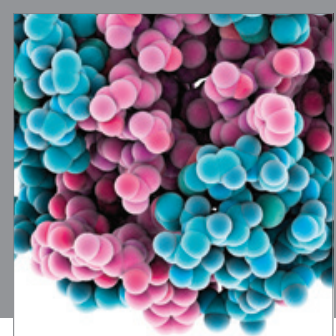

Journal of
Diabetes Research

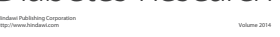

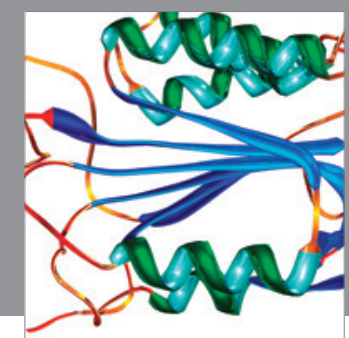

Disease Markers
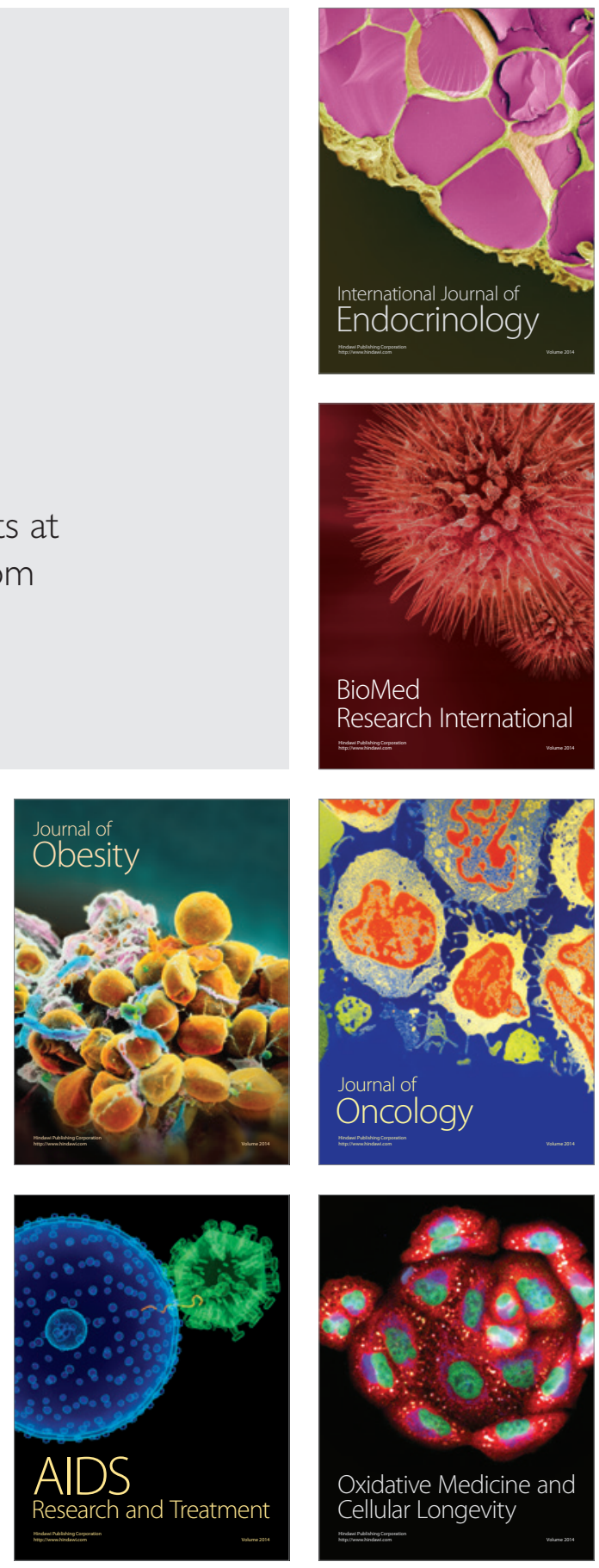